INVADER ATTRIBUTES

Allelopathy

Apomixis

Demography

Dispersal Ability, Animal

Dispersal Ability, Plant

Genotypes, Invasive

Geographic Origins and Introduction Dynamics

Invasiveness

Life History Strategies

Mutualism

"Native Invaders"

Novel Weapons Hypothesis

Reproductive Systems, Plant

Seed Ecology

Taxonomic Patterns

Tolerance Limits, Animal

Tolerance Limits, Plant

Vegetative Propagation

\section{ECOSYSTEM FEATURES}

Belowground Phenomena

Disturbance

Eutrophication, Aquatic

Habitat Compatibility

Invasibility, of Communities and Ecosystems

Islands
Lakes

Nitrogen Enrichment

Protected Areas

Rivers

Seas and Oceans

Succession

Wetlands

\section{PROCESSES}

Climate Change

Enemy Release Hypothesis

Epidemiology and Dispersal

Evolutionary Response, of Natives to Invaders

Evolution of Invasive Populations

Fire Regimes

Hybridization and Introgression

Invasional Meltdown

Lag Times

Land Use

Propagule Pressure

Range Modeling

Succession

\section{IMPACTS}

Allelopathy

Competition, Animal

Competition, Plant 
Endangered and Threatened Species

Fire Regimes

Herbivory

Hybridization and Introgression

Hydrology

Invasion Economics

Landscape Patterns of Plant Invasions

"Native Invaders"

Novel Weapons Hypothesis

Pollination

Transformers

Weeds

NOTABLE TAXA

Algae

Ants

Bees

Birds

Brown Treesnake

Bryophytes and Lichens

Burmese Python and Other Giant Constrictors

Carnivores

Carp, Common

Cheatgrass

Crabs

Crayfish

Crustaceans (Other)

Disease Vectors, Human

Earthworms

Eucalypts

Fishes

Flaviviruses

Forest Insects

Freshwater Plants and Seaweeds

Fungi

Grasses and Forbs

Grazers

Gypsy Moth

Hemlock Woolly Adelgid

Influenza
Invertebrates, Marine

Kudzu

Ladybugs

Lantana camara

Malaria Vectors

Mammals, Aquatic

Melastomes

Mosquitoes

Mycorrhizae

Nile Perch

Parasites, of Animals

Parasitic Plants

Pathogens, Animal

Pathogens, Human

Pathogens, Plant

Phytophthora

Predators

Rats

Reptiles and Amphibians

Rinderpest

Rodents (Other)

Sea Lamprey

Small Indian Mongoose

Snails and Slugs

Trees and Shrubs

Vines and Lianas

Wasps

Water Hyacinth

Zebra Mussel

PATHWAYS TO INVASION

Acclimatization Societies

Agriculture

Aquaculture

Aquaria

Ballast

Canals

Forestry and Agroforestry

Game Animals

Horticulture 
Ostriculture

Pet Trade

\section{MANAGEMENT AND REGULATION}

Agreements, International

Biological Control, of Animals

Biological Control, of Plants

Black, White, and Gray Lists

CART and Related Methods

Databases

Early Detection and Rapid Response

Ecoterrorism and Biosecurity

Eradication

Herbicides

Integrated Pest Management

Laws, Federal and State

Mechanical Control

Pesticides (Fish and Mollusc)

Pesticides for Insect Eradication

Pesticides (Mammal)

Range Modeling
Regulation (U.S.)

Remote Sensing

Restoration

Risk Assessment and Prioritization

HISTORY

DAISIE Project

Darwin, Charles

Elton, Charles $S$.

Invasion Biology

Invasion Biology: Historical Precedents

SCOPE Project

Xenophobia

\section{NOTABLE INVASIONS}

Australia: Invasions

Great Lakes: Invasions

Hawaiian Islands: Invasions

Mediterranean Sea: Invasions

New Zealand: Invasions

Ponto-Caspian: Invasions

South Africa: Invasions 
This page intentionally left blank 